\title{
WAKEFIELD EXCITATION IN MULTIMODE STRUCTURES BY A TRAIN OF ELECTRON BUNCHES*
}

\author{
J.G. POWER*, M.E. CONDE, W. GAI, R. KONECNY, and P. SCHOESSOW \\ Argonne National Laboratory, Argonne, Illinois 60439
}

\section{Abstract}

We discuss wakefield excitation and propagation in dielectric structures, particularly concentrating on the case of multiple drive beam excitation in multimode structures. We emphasize calculations of the energy loss of the drive bunch train, the amplitude of the wakefield, and the relation between power flow and stored energy in the dielectric wakefield device. We show that for a collinear multimode structure the amplitude of the wakefield generated by a bunch train is less than or equal to the wakefield generated by a single bunch of the same total charge. Furthermore, the transformer ratio, $R$, is shown to be always less than 2, even in the multiple drive beam case. Plans for an experiment to measure wakes in a multimode structure at AWA are presented.

\section{INTRODUCTION}

The efficiency of a collinear wakefield accelerator scheme, i.e. one for which the drive and witness beams follow identical trajectories through the accelerator system is limited by the transformer ratio $R$. Under very general assumptions $R<2$, where $R$ is defined as the ratio of the maximum energy gain behind the drive bunch to the maximum energy loss inside the drive bunch [2]. There have been a number of techniques studied to enhance the transformer ratio by violating one or more assumptions of the wakefield theorem: asymmetric drive beams [3], noncollinear drive/test beams [4] and more $[5,6]$.

A recent article [1] has described a multimode dielectric structure driven by a bunch train. The authors make two claims. One, a small transformer ratio enhancement for the single bunch case is obtained through the use of a superposition of multiple modes. Two, a larger effect (than expected from linear scaling with total drive charge) is predicted in the case of multiple drive bunches and attributed to a process described as "stimulated emission" of Cherenkov radiation. We have reanalyzed the problem of multimode structure wakefields using the same assumptions as the authors of reference [1]. We have concluded that there is no enhancement in the transformer ratio beyond that expected in the linear theory. Multimode dielectric structures, however, are of some scientific interest. We are currently preparing an experiment at the Argonne Wakefield Accelerator [7] to study wakefields produced by a bunch train in one of these devices."

\section{MULTIPULSE, MULTIMODE DEVICES}

\subsection{Direct Solution of the Wave Equation}

One can determine the wakefield excitation of any cylindrically symmetric structure [9] by first finding the point charged particle (Green Function) solution,

$$
G_{z}(r, z)=\sum_{n=1}^{\infty} G_{n}(r) \cos \left(k_{n} z\right)
$$

For a series of $M$ bunches separated by a distance, $\lambda$ "bunch train") the charge distribution can be expressed as.

$$
f(z)=\sum_{m=1}^{M} f_{m}(z-m \lambda)
$$

The total wakefield excited by a bunch train at any point $\mathrm{z}$ (which may be inside a bunch) is obtained by taking the convolution of the Green function [1] over the charge distribution [2],

$$
\begin{aligned}
W_{z}(r, z)= & \left.\int_{-\infty}^{z} \sum_{m=1}^{M} f_{m}(z-m \lambda) \backslash \sum_{n=1}^{\infty} G_{n}(r) \cos \left[\left(k_{n} z-z^{\prime}\right)\right]\right\} d z^{\prime} \\
& \equiv \sum_{m=1}^{M} W_{z m}(r, z)
\end{aligned}
$$

\subsection{Conservation of Energy}

For the $m^{\text {th }}$ bunch in the train, the energy loss per unit distance traveled $\left(U_{m}\right)$ can be expressed as,

$$
\begin{aligned}
U_{m} & =\int_{-\infty}^{\infty} d z f_{m}(z-m \lambda) \int_{-\infty}^{z} d z^{\prime} f_{m}(z-m \lambda) G\left(r, z-z^{\prime}\right) \\
& +\int_{-\infty}^{\infty} d z f_{m}(z-m \lambda) \sum_{m=1}^{m-1} W_{z m}(r, z)
\end{aligned}
$$

In (4) the total energy loss is comprised of two terms: that due to the $\mathrm{m}^{\text {th }}$ bunch (first term) and that due to the previous $\mathrm{m}-1$ bunches (second term). The stored energy

$$
\begin{gathered}
\text { per unit length }\left\{\frac{1}{2 L} \int_{z}^{2+L} d z \int_{s} d s\left(\varepsilon \vec{E}^{2}+H_{\varphi}{ }^{2}\right)\right\} \text { is } \\
U=K\left(\sum_{m=1}^{M} \sum_{n=1}^{\infty} \eta_{n m} E_{n}\right)^{2}=K\left(\sum_{m=1}^{M} W_{m}\right)^{2}
\end{gathered}
$$

where $\eta_{\mathrm{mm}}$ is a form factor depending on the detailed bunch distribution, $\mathrm{W}_{\mathrm{m}}$ is the peak wakefield left behind by the $\mathrm{m}^{\mathrm{t}}$ bunch and $\mathrm{K}$ is a geometric factor that can be determined either through direct integration or by the method shown in the following section.

\footnotetext{
'This work is supported by DOE contract

"Email: jp@hep.anl.gov
}

To be presented at the 1999 Particle Accelerator Conference (PAC'99) New York, New York, March 29 - April 02, 1999. 


\section{DISCLAIMER}

This report was prepared as an account of work sponsored by an agency of the United States Government. Neither the United States Government nor any agency thereof, nor any of their employees, make any warranty, express or implied, or assumes any legal liability or responsibility for the accuracy, completeness, or usefulness of any information, apparatus, product, or process disclosed, or represents that its use would not infringe privately owned rights. Reference herein to any specific commercial product, process, or service by trade name, trademark, manufacturer, or otherwise does not necessarily constitute or imply its endorsement, recommendation, or favoring by the United States Government or any agency thereof. The views and opinions of authors expressed herein do not necessarily state or reflect those of the United States Government or any agency thereof. 


\section{DISCLAIMER}

Portions of this document may be illegible in electronic image products. Images are produced from the best available original document. 
By energy conservation, the stored energy per unit length (5) is equal to the sum of the energy loss by each bunch (4) plus the energy flow (P) of the wave.

$U=K\left(\sum_{m=1}^{M} W_{m}\right)^{2}=\sum_{m=1}^{M} U_{m}+\sum_{m=1}^{M} \frac{P_{m}}{c}=\sum_{m=1}^{M} U_{m}\left(1+\frac{v_{g}}{c}\right)$

where the result $U_{m}=P_{m} / v_{g}[10]$ has been used.

Using (4) to calculate the energy loss of the first bunch, one can use (6) to calculate the wakefield of subsequent bunches (as done in ref. [1]). However, one has to evaluate the right hand side of (6) carefully, because $U_{m}$ depends not only on the self wakefield, but also on the wakefield generated by previous bunches; more importantly, it has strong dependence on the detailed distribution of the $\mathrm{m}^{\text {th }}$ bunch.

\subsection{Gaussian Bunch Train Example}

We consider wakefields generated by a train of $M$ gaussian bunches in a perfectly harmonic structure (i.e. where the modes are equally spaced in frequency).

$$
f(z)=\frac{N}{(2 \pi)^{1 / 2} \sigma_{z}} \sum_{m=1}^{M} e^{-\frac{(z-m \lambda)^{2}}{2 \sigma_{z}^{2}}}
$$

The energy loss by the $1^{\text {st }}(\mathrm{m}=1)$ bunch (4) is

$$
U_{1}=\frac{1}{2} \sum_{n=1}^{\infty} G_{n} \exp \left(-\left(k_{n} \sigma_{z}\right)^{2}\right.
$$

The wakefield behind this bunch $\left(z \gg \sigma_{z}\right)$ is then easily obtained (3) as

$$
W_{1}(z)=\sum_{n=1}^{\infty} G_{n} \exp \left(-\frac{\left(k_{n} \sigma_{z}\right)^{2}}{2}\right) \cos k_{n} z
$$

For a perfectly harmonic structure, at $z=\lambda, \cos \left(\mathrm{k}_{\mathrm{n}} \mathrm{z}\right)=1$. Therefore, the maximum wakefield left behind at $z=\lambda$ is,

$$
W_{1}=\sum_{n=1}^{\infty} G_{n} \exp \left(-\frac{\left(k_{n} \sigma_{z}\right)^{2}}{2}\right)
$$

Using equation 6 , we find $K$ as

$$
K=\frac{\frac{1}{2} \sum_{n=1}^{\infty} G_{n} \exp \left(-\left(k_{n} \sigma_{z}\right)^{2}\right.}{\left[\sum_{n=1}^{\infty} G_{n} \exp \left(-\frac{\left(k_{n} \sigma_{z}\right)^{2}}{2}\right)\right]^{2}}\left(1+\frac{v_{g}}{c}\right)
$$

Now we calculate the wake amplitude after the $2^{\text {nd }}$ bunch. The energy loss of the second beam, $\mathrm{U}_{2}$, is

$$
U_{2}=\frac{1}{2} \sum_{n=1}^{\infty} G_{n} \exp \left(-\left(k_{n} \sigma_{z}\right)^{2}\right)+\sum_{n=1}^{\infty} G_{n} \exp \left(-\left(k_{n} \sigma_{z}\right)^{2}\right)
$$

Solving equation 6 for two bunches we now have $K\left(W_{1}+W_{2}\right)^{2}=\left(U_{1}+U_{2}\right)\left(1+v_{g} / c\right)$. Using this result and equation 12 we have, $W_{1}+W_{2}=2 W_{1}$ and therefore,

$$
W_{2}=W_{1}
$$

In the same way, one can prove

$$
W_{2}=W_{3}=\cdots=W_{M}
$$

From above calculation, we conclude that the wakefield can be calculated using linear superposition (convolution of the Green function for a point particle over the charge distribution of the multiple bunches) or by conservation of energy. Both methods yield identical results; there is no transformer ratio enhancement in either the single or multiple bunch multimode devices.

\section{DESIGN OF A MULTIMODE DIELECTRIC STRUCTURE}

A multiple beam, multimode dielectric wakefield accelerator (M-DWFA) is designed for an experiment at the AWA using the analytic results for dielectric structure derived in reference [9]. The structure is a cylindrical dielectric waveguide $(\varepsilon=38.1)$ with an inner radius 0.5 $\mathrm{cm}$, outer radius $1.44 \mathrm{~cm}$ and $60 \mathrm{~cm}$ in length. The AWA can transport a $10 \mathrm{nC}$ beam through a $60 \mathrm{~cm}$ long tube with a $1 \mathrm{~cm}$ diameter hole. For the multiple beam experiment at AWA, the acceleration wavelength is designed to be $23.05 \mathrm{~cm}$ since $\lambda_{\mathrm{rf}}$ is $23.05 \mathrm{~cm}$ at $1.3 \mathrm{GHz}$. Successive drive bunches can then be located one fundamental wavelength apart in the M-DWFA.

\section{EXPERIMENTAL SETUP}

The AWA drive linac is configured to provide a collinear bunch train of 4 bunches, each with charge $\mathrm{Q}=10 \mathrm{nC}$, bunch length, $\sigma_{z}=2 \mathrm{~mm}$ and energy spread $\sigma_{\varepsilon} / \mathrm{E}=1 \%$. Each bunch must be placed so that the individual wakes left by each bunch adds constructively to the wake of the preceding bunch. For our experimental configuration, simulation show that the spacing between bunches must be accurate to within $1 \mathrm{~mm}$ out of $230.5 \mathrm{~mm}$ for appreciable constructive interference to take place.

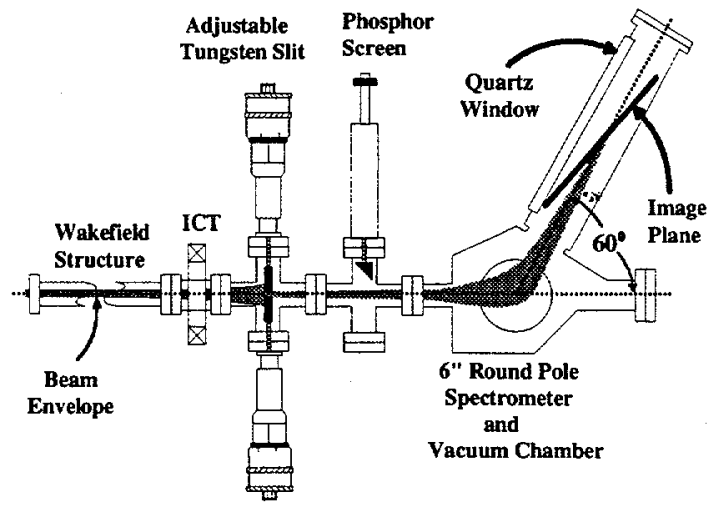

Figure 1: Energy Measurement System. The decelerated drive bunch train passes through a Tungsten slit, which is imaged through a $60^{\circ}$ bend onto a phosphor screen.

This fact places a high demand on the AWA's energy measurement system. A new imaging spectrometer was developed [Fig. 1] to provide improved momentum 
resolution over a wider range while reducing the sensitivity to beam jitter.

\section{SIMULATION OF THE EXPERIMENT}

Before a witness beam can be accelerated, the 4 drive beams must be properly positioned. To this end, we designed an experiment to accurately measure the energy spectrum of the 4 drive beams. Using the method of [9] and the expression for the wakefield of a bunch train from [8] the computed wake potential as a function of $\mathrm{z}$ (the distance behind the first bunch) is shown

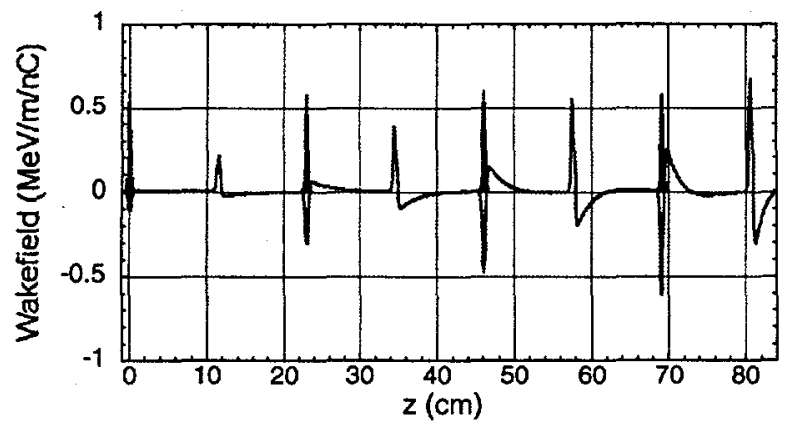

Figure 2: The wake excited by 4 monochromatic drive beams. (Beam moving right to left.)

in Fig. 2. Using the results displayed in Fig. 2 one may easily extract the energy spectrum generated by the 4 monochromatic drive beams. Projecting the wake experienced by each particle within the bunch onto the energy axis and scaling by the appropriate values of $L$ and $Q$ gives this spectrum. The total energy spectrum is then computed by taking the convolution of the monochromatic energy spectrum with the drive bunch's energy distribution function, which is measured to be approximately Gaussian in shape. For tube length $\mathrm{L}=59.87 \mathrm{~cm}$, charge $\mathrm{Q}=10 \mathrm{nC}$, energy spread $\sigma_{\mathrm{E}} / \mathrm{E}=1 \%$ and initial drive beam energy of $14 \mathrm{MeV}$ we plot the total energy spectrum in Fig. 3.

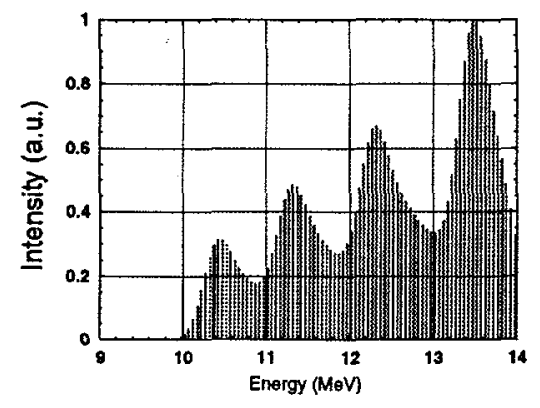

Figure 3: Energy spectrum of bunch train after exiting the multimode structure

The multimode structure may have tighter manufacturing tolerances than the single mode device because of the requirement that the various modes constructively interfere at the appropriate delays corresponding to the spacing of the bunch train. An error in either $\varepsilon$ or the geometry leads to a change in the accelerating wavelength. We examine as typical the case of a maximum deviation from the nominal value of the permittivity. Given the vendor's specification of $\varepsilon=38.1 \pm 2 \%$ we calculate the wakefield excited by the same bunch train as above but with $\varepsilon=37.34$ (38.86) corresponding to $-2 \%$ $(+2 \%)$ out of tolerance. This results in a shortening (lengthening) of the acceleration wavelength by $2.5 \mathrm{~mm}$ $(1.5 \mathrm{~mm})$. The effect of this is that the drive bunches arrive late in the structure causing the wakefields of the individual bunches to begin to destructively interfere with each other. However, the bunch train can easily be adjusted to accommodate the errant wavelength.

A more serious problem is that $\varepsilon$ may not be constant over the frequency range excited causing the nearly harmonic spectrum and hence the constructive interference to be disrupted.

\section{SUMMARY}

We have calculated the wakefields in multimode dielectric structures excited by a bunch train. We found no enhancement to the transformer ratio when a single beam drives this structure. We found no wakefield enhancement for this device over a single beam with the same total charge as the drive train. Our calculations show that the method of linear superposition and the energy conservation method both predict the same wakefields. This type of wakefield device may still have good breakdown properties due to its relatively short $\mathrm{rf}$ pulse.

\section{REFERENCES}

[1] T-B. Zhang, J. L. Hirshfield, T. C. Marshall, B. Hafizi, Phys. Rev. E56 4647 (1997)

[2] P. B. Wilson, Proc. of the 13th SLAC Summer Inst. on Particle Physics, SLAC Report No.296, p. 273, E. Brennan ed., (1985)

[3] K.L. Bane, P. Chen, P.B. Wilson, IEEE Trans. Nucl. Sci. 323524 (1985)

[4] P. Schoessow, M. E. Conde, W. Gai, R. Konecny, J. Power, J. Simpson, J. Appl. Phys 84663 (1998)

[5] A. G. Ruggiero, P. Schoessow, J. Simpson, Proc. of Symp. on Advanced Accelerator Concepts, Madison, WI, Aug 21-27, 1986, F. Mills ed., AIP Press (1986)

[6] J. Rosenzweig, P. Schoessow, B. Cole, W. Gai, R. Konecny, J. Norem, J. Simpson, Phys. Rev. A39 1586 (1989)

[7] URL: http:/www.hep.anl.gov/awa/awa/awahome.htm

[8] J.G. Power, W. Gai, P. Schoessow, submitted to Phys. Rev. E. A preprint is also available on the web:

http://www.hep.anl.gov/awa/awa/pubs.htm.

[9] M. Rosing, W. Gai, Phys. Rev. D42 1829 (1990)

[10]J.D.Jackson, Classical Electrodvnamics. I.Wilev 1974

The submitted manuscript has been created by the University of Chicago as Operator of Argonne National Laboratory ("Argonne") under Contract No. W-31-109-ENG-38 with the U.S. Department of Energy. The U.S. Government retains for itself, and others acting on its behalf, a paid-up, nonexclusive, irrevocable worldwide license in said article to reproduce, prepare derivative works, distribute copies to the public, and pertorm publicly and display publicly, by or on behalf of the Government. 